\title{
Evidence for gene-smoking interactions for hearing loss and deafness in Japanese American families
}

\author{
Jia Y. Wan ${ }^{\text {a }}$, Christina Cataby ${ }^{\mathrm{b}}$, Andrew Liem ${ }^{\mathrm{a}}$, Emily Jeffrey ${ }^{\mathrm{a}}$, \\ Trina M. Norden-Krichmar ${ }^{a}$, Deborah Goodman ${ }^{a}$, Stephanie A. Santorico ${ }^{c}$, \\ Karen L. Edwards a, b, *, American Diabetes Association GENNID Study Group \\ a Department of Epidemiology, University of California, Irvine, United States \\ ${ }^{\mathrm{b}}$ Department of Population Health and Disease Prevention, University of California, Irvine, United States \\ c Department of Mathematical and Statistical Sciences, University of Colorado, Denver, United States
}

\section{A R T I C L E I N F O}

Article history:

Received 19 September 2019

Received in revised form

5 December 2019

Accepted 18 December 2019

Available online 24 December 2019

Keywords:

Gene-environment interaction

Genome-wide linkage

Hearing loss

Smoking

Family

Linkage

\begin{abstract}
A B S T R A C T
Background: This study investigated the relationship between smoking and hearing loss and deafness (HLD) and whether the relationship is modified by genetic variation. Data for these analyses was from the subset of Japanese American families collected as part of the American Diabetes Association Genetics of Non-insulin Dependent Diabetes Mellitus study. Logistic regression with generalized estimating equations assessed the relationship between HLD and smoking. Nonparametric linkage analysis identified genetic regions harboring HLD susceptibility genes and ordered subset analysis was used to identify regions showing evidence for gene-smoking interactions. Genetic variants within these candidate regions were then each tested for interaction with smoking using logistic regression models. Results: After adjusting for age, sex, diabetes status and smoking duration, for each pack of cigarettes smoked per day, risk of HLD increased 4.58 times (odds ratio $(O R)=4.58 ; 95 \%$ Confidence Interval $(\mathrm{CI})$ : $(1.40,15.03))$, and ever smokers were over 5 times more likely than nonsmokers to report HLD $(O R=5.22 ; 95 \% \mathrm{CI}:(1.24,22.03))$. Suggestive evidence for linkage for HLD was observed in multiple genomic regions (Chromosomes 5p15, 8p23 and 17q21), and additional suggestive regions were identified when considering interactions with smoking status (Chromosomes 7p21,11q23,12q32, 15q26, and 20q13) and packs-per-day (Chromosome 8q21).

Conclusions: To our knowledge this was the first report of possible gene-by-smoking interactions in HLD using family data. Additional work, including independent replication, is needed to understand the basis of these findings. HLD are important public health issues and understanding the contributions of genetic and environmental factors may inform public health messages and policies.
\end{abstract}

\section{Introduction}

In the United States, hearing loss is the most common sensory impairment affecting about 1 in every 8 Americans and is the most prevalent sensory disorder in developed countries (Lin et al., 2011a; Uchida et al., 2012). Furthermore, Americans with diabetes are at an increased risk of hearing loss (Bainbridge et al., 2008), with evidence that Japanese with diabetes are twice as likely to have hearing loss (Horikawa et al., 2013). Severe to profound hearing loss

\footnotetext{
* Corresponding author. 222 Irvine Hall, University of California, Irvine, Irvine, 92697, CA, United States.

E-mail address: kedward1@uci.edu (K.L. Edwards).
}

is expected to cost society $\$ 297,000$ over the lifetime of an individual, with most of those losses due to reduced work productivity (Mohr et al., 2000). Typical risk factors for acquired hearing loss are trauma to the head and ear, excessive noise exposure (occupational and recreational), and aging (Mayo Clinic, 2015). Additionally, several studies have demonstrated that smoking is positively associated with risk of hearing loss disorder (HLD) (Agrawal et al., 2008; Siegelaub et al., 1974; Nomura et al., 2005), especially hearing loss at high frequencies with the attenuation of the association after smoking cessation among Japanese (Hu et al., 2019): however, the results are not consistent (Karlsmose et al., 2000; Lin et al., 2011b). One potential explanation for these inconsistent findings is that the relationship between smoking and HLD may be modified 
by other factors, including underlying genetic variation.

More than $50 \%$ of prelingual deafness, defined as hearing loss which begins prior to the onset of speaking, is genetic, most often autosomal recessive, and not associated with other physical symptoms (nonsyndromic) (Ito et al., 2010). While acquired hearing loss is typically attributed to environmental factors, genetic factors also play a role. For example, family history is significantly associated with hearing loss (Smith et al., 2014; McMahon et al., 2008), and approximately $36 \%$ of the variance is attributable to genetic variation $\left(h^{2}=0.36\right)$ (Kvestad et al., 2012). However, the genetic transmission of postlingual hearing impairment is predominantly autosomal dominant (Shearer et al., 2014). In a familybased study, Huyghe and colleagues (Huyghe et al., 2008) identified a linkage peak at $8 \mathrm{q} 24.13-\mathrm{q} 24.22$, representing the first locus identified for an age-related hearing impairment trait. Furthermore, O'Neil et al. (O'Neill, 1996) performed linkage analysis on a large multi-generational family in which a gene for autosomal dominant late-onset progressive nonsyndromic hearing impairment was segregating, and identified DNFB10 on Chromosome 6q. Lavinsky and colleagues (Lavinsky et al., 2015) conducted a genome-wide association study (GWAS) and identified NADPHoxidase 3, located on chromosome 6, as a critical gene for susceptibility to hearing loss. In another GWAS, Friedman and researchers (Friedman et al., 2009) identified common alleles of GRM7, located on Chromosome 3, that contribute to an individual's susceptibility of developing age-related hearing impairment. To date, over two dozen genes have been identified for autosomal recessive nonsyndromic hearing loss (Kvestad et al., 2012; Hilgert et al., 2009; Dickson et al., 2010). Moreover, among the Japanese, certain genes were identified as 1) being causal: GJB2 on chromosome 13q12.11, mitochondrial DNA 12SrRNA; 2) having an association with type 2 diabetes (T2D) and audiovestibular phenotypes (Rohayem et al., 2011): WFS1 on chromosome 4p16.1 (Kobayashi et al., 2018); and 3 ) being related to abnormal enlargement of the vestibular aqueduct in the inner ear: SLC26A4 on chromosome 7q22.3, EYQ1, and SIX1 on chromosome 14q23.1 (Ito et al., 2010).

\section{Theory}

While both environmental and genetic factors are involved in HLD, to our knowledge, interactions have not been investigated, particularly those between smoking and genetic susceptibility to HLD. Therefore, the main purpose of this study was to investigate this question using existing data from a sample of Japanese American families recruited or studies of T2D.

\section{Methods and methods}

\subsection{Study subjects}

From 1993 to 2003, the American Diabetes Association established the GENNID (Genetics of Non-insulin Dependent Diabetes Mellitus) study, a multicenter family-based resource to identify the genetic components of Type 2 Diabetes (T2D) (Raffel et al., 1996). The proportion of those with HLD was significantly different across the GENNID ethnic groups $\left(\chi_{\mathrm{df}=3}^{2}=22.65\right.$, $\mathrm{p}$-value $\left.=4.78 \times 10^{-5}\right)$ with $7.4 \%$ HLD in African Americans, 17.1\% HLD in European Americans, 23.3\% HLD in Japanese Americans, and 13.9\% HLD in Mexican Americans. Because the greatest prevalence of HLD was seen among Japanese Americans families, our study focused on this group.

\subsection{Data collection}

Extensive data were collected as described previously (Ehm et al., 2000), including self-reported information on HLD and age of deafness. To determine HLD status, participants were asked "Have you been told by a health care worker that you have hearing loss/deafness?" Information on smoking, including ever smoking cigarettes, age began smoking, age stopped smoking, and packsper-day were also self-reported. In particular, smoking characteristics of interest were ever-smoking and packs-per-day, although differences in duration of smoking and age at smoking were also evaluated between non-HLD and HLD subjects. Moreover, each pack of cigarettes can be roughly quantified as 20 cigarettes as standard in the U.S. at the time of data collection.

According to guidelines from the World Health Organization (WHO, 2006), diabetes status was defined as having a 2-hour plasma glucose $\geq 200 \mathrm{mg} / \mathrm{dl}$ (11.1 mmol/l) during an OGTT. The genetic data consisted of a genome-wide linkage panel with 374 polymorphic microsatellite markers yielding average marker spacing of approximately $9.42 \mathrm{cM}$ and median marker spacing of $9.10 \mathrm{cM}$. Descriptions of the genetic data cleaning and pedigree checking have been summarized previously (Edwards et al., 2008). The microsatellite markers were used in the genome-wide linkage analysis and OSA. In addition, all samples were also genotyped using Illumina's Multi-Ethnic Global BeadChip (v1.0). After quality control, imputation was also performed after phasing using the 1000 Genomes Phase 3 panel and was corrected for relatedness (unpublished). Both imputed and genotyped single nucleotide variants (SNVs) were available for evaluating gene-by-environment (GxE) interaction under linkage regions of interest.

There were a total of 17 pedigrees, of which 15 families had at least one HLD case. However, because we were interested in evaluating GxE, we selected those families with at least two biologically related cases of HLD reported in the pedigree to increase the likelihood of underlying genetic influences. Eight families with a total of 97 family members met this criterion, of which 75 members had genetic and phenotypic data available for our analyses. These eight families were used for determining candidate genetic regions with GxE among subsets of families using the ordered subset analysis (OSA) method on genome-wide microsatellite linkage scan results. In order to follow-up within these candidate regions, all Japanese American GENNID families ( $\mathrm{N}=121$ samples) were used to test for GxE at imputed and genotyped SNVs.

\subsection{Statistical analysis}

We first evaluated the association between smoking and HLD. HLD was coded as a binary "yes/no" variable for all analyses. Logistic regression models were used with generalized estimating equations (GEE) and independence correlation structure to account for between-family-member correlations. Two sets of adjusted models for each smoking variable (ever-never smoking and packsper-day) were examined. Model 1 was adjusted for age, sex, and diabetes status. Model 2 included all the covariates included in Model 1, plus duration of smoking, calculated as the difference in age stopped smoking and age began smoking. Odds ratios (ORs) and their corresponding 95\% Confidence Intervals (CIs) were also calculated and shown. Non-smokers had a smoking duration of 0 years. Statistical significance was based on the Wald's Z-test, and p-values $<0.05$ were considered statistically significant. Analyses were performed using the gee package (Carey, 2015) in R software version 3.2.5 (Team, 2015).

Next, we performed a genome-wide linkage scan for HLD susceptibility regions using a nonparametric two-point linkage (NPL) analysis at each marker (Whittemore and Halpern, 1994; Kong and Cox, 1967) with the Cox and Kong exponential model. Because we were looking genome-wide, we used a non-parametric model as this approach makes the fewest assumptions regarding mode of 
inheritance. Analyses were based on data from 374 microsatellite markers across the genomes of 75 subjects from the eight families with at least two HLD cases. The NPL routine in MERLIN software (Abecasis et al., 2002) was used. LOD scores greater than 3 and 1.9 provide significant and suggestive evidence of linkage, respectively.

Next, ordered subset analysis (OSA) (Hauser et al., 2004) was implemented to identify those chromosomal regions with evidence for differences in linkage by smoking status (i.e., GxE). That is, in the presence of etiologic heterogeneity based on smoking, we located chromosomal regions that have maximal evidence of linkage with a subset of families based on smoking. Here, a genome-wide OSA was conducted at each marker as implemented in FLOSS software (Browning, 2006). Based on prior studies, smoking appears to increase risk of HLD; hence, we used a one-sided test. Specifically, families were ranked in descending order (high to low) using perfamily summary values of the given smoking covariate (i.e., percentage of ever smokers in the family or average packs-per-day smoked among family members). Then, families were added oneat-a-time in descending smoking order into the two-point NPL analysis until a maximum resulting LOD (LOD-OSA) was achieved at each marker. P-values were calculated as the proportion of random family rankings that gave permuted LOD-OSA scores greater than the original LOD-OSA using a permutation test. At least 100 random rankings were needed to adequately determine the $\mathrm{p}$-value by the Besag-Clifford sequential stopping rule (Besag and Clifford, 1991). Significance of the gene-by-smoking interaction in the subset of families is determined if the OSA p-value $<0.002$ which corresponds to a Bonferroni correction for separate tests on 22 chromosomes (i.e., 0.05/22). Furthermore, because our results are exploratory, suggestive evidence of GxE interaction is determined if the OSA p-value $<0.05$ per chromosome.

Finally, logistic regression models using GEE were used to evaluate individual SNVs for GxE within the putative genetic regions identified by OSA. The independence correlation structure in GEE was used to adjust for within family correlations, to maximize power as well as reduce type I error and bias in OR estimation (Hancock et al., 2007). Analyses using the entire JapaneseAmerican GENNID sample ( $\mathrm{N}=121$ subjects) were adjusted for age, sex, and diabetes status, and were performed using the GWAF package (Chen and Yang, 2015) in R (R Core Team, 2019). To correct for the multiple testing of 311,989 SNVs in 6 candidate regions (Table 4), the conservative Bonferroni method was used to adjust the significance threshold in which p-values less than $1.6 \times 10^{-7}$ (i.e., 0.05/311,989) were considered as significant evidence of GxE interaction. All results were converted in the human genome from GrCh37/hg19 to build GrCh38/hg38 using the LiftOver tool (Hinrichs et al., 2006) and then annotated using SeattleSeq version 151 (Ng et al., 2009), and interaction p-value plots of select candidate regions were made using Locus Zoom (Pruim et al., 2010).

\section{Results}

The characteristics of 75 Japanese American individuals in 8 families are presented in Table 1 ; there was a median of 10 subjects per family with a minimum of 9 subjects and a maximum of 19 subjects per family. Individuals with HLD were significantly older than those without HLD ( $67 \pm 11$ years compared to $51 \pm 15$ years, respectively; $\left.\mathrm{p}=1.23 \times 10^{-9}\right)$. The total sample was $53 \%$ female, and there was a significantly higher proportion of women among HLD cases compared to non-HLD individuals. Specifically, $71 \%$ of HLD cases were female compared to $45 \%$ of non-HLD individuals $(P=0.030)$. Additionally, there was evidence of increased smoking exposure in the HLD group compared to the non-HLD group $(P=0.001)$, longer duration of cigarette smoking $(P=0.014)$, and higher average packs-per-day $(P=0.049)$.
Table 2 describes demographic characteristics for each of the eight families, which are ordered based on decreasing percentages of ever-smokers per-family. Families differed significantly by age (mean ranging from 47.57 years to 67.31 years) and gender (at least $50 \%$ of five of eight families were predominantly female). In addition, smoking characteristics differed significantly among families, with the number of ever smokers ranging from $86 \%$ to $18 \%$, the duration of smoking ranging from 29.33 years to 2.82 years, and the number of packs-per-day smoked ranging from 1.17 packs-per-day to 0.05 packs-per-day.

In all logistic regression models, there was a significant and positive relationship between HLD and both ever-smoking and packs per-day (Table 3). Being an ever-smoker increased the odds of HLD by 5.0- fold (unadjusted), 2.83-fold when adjusted for age, sex and history of diabetes (Model 1), and 5.76-fold when additionally adjusted for smoking duration (Model 2). Furthermore, for every additional pack-per-day smoked, the odds of HLD increased 2.04fold (unadjusted) yet increased 4.97-fold after adjusting for age, sex, diabetes, and smoking duration (Model 2).

Results from the nonparametric linkage analysis indicated suggestive evidence for linkage to putative HLD loci (LOD >1.9) for three chromosomal regions (Supplemental Fig. 1, grey line). On chromosome 5p15.3 at D5S2505 (13.6 cM), the LOD was 2.18, while on 8 p23.1 at D8S277 (11.3 cM), the LOD was 2.19. The highest evidence for linkage was found on chromosome 17q21.3 at ATC6A06 $(68.3 \mathrm{cM})$ where the LOD was 2.31 .

Table 4 and Supplemental Fig. 1 (blue, red, and magenta lines) show the regions of the strongest evidence for gene-environment interaction in the families with the highest per-family percentage of smoking and average packs-per-day. Analyzing the top 4 families ranked by their average packs-per-day values, chromosome $8 \mathrm{q} 21$ at D8S1119 $(89 \mathrm{cM})$ had an OSA max LOD score of $3.54(P=0.04)$, providing evidence for gene-smoking interaction nearby the HLD suggestive linkage region ( $\mathrm{LOD}=2.19$ at $11.3 \mathrm{cM}$ ). These four families (Families 1,2,3,5 in Table 2) also had the highest duration of smoking, highest percentage of ever smoking, and higher prevalence of T2D. The other GxE regions based on OSA p-values of less than 0.05 were not in the proximity of regions showing suggestive evidence for linkage to HLD (Supplemental Fig. 1). The OSA max LOD score of $2.9(P=0.01)$ and $2.6(P=0.01)$ for the top 5 and top 3 ranked families based on percentage of smokers supports a geneenvironment interaction on chromosome $15 \mathrm{q} 26$ at D15S816 $(96.4 \mathrm{cM})$ and on chromosome $12 \mathrm{q} 32$ at D12S1045 (145.8 cM), respectively. Additionally, on chromosome 20q13 at D20S171 $(92.2 \mathrm{cM})$ there was also evidence of GxE using OSA with the top 3 families based on percentage of ever smoking yielding an OSA LOD score of $3.03(P=0.02)$. Furthermore, there was evidence of geneby-smoking interaction for chromosomes 7p21 $(P=0.04)$ and $11 \mathrm{q} 23(P=0.03)$ in the top 2 families based on percentage of ever smoking.

Evaluating GxE among the single nucleotide variants (SNVs) underlying the candidate regions in Table 4, there was evidence of hearing loss risk associated with SNVs on chromosome 8 being modified by smoking. The 23 variants that significantly modified the association between hearing loss and packs per day are summarized in Supplementary Table 1. In particular, intronic SNVs within PDE7A, MTFR1 (Fig. 1), ZBTB10 and PAG1 genes (Supplementary Fig. 2) as well as novel intergenic SNVs were shown to interact with smoking on the risk of HLD.

\section{Discussion}

While it is well-established that smoking is harmful to one's health, our findings lend support to the observation that smoking is related to HLD based on this sample of Japanese American families. 
Table 1

Demographic characteristics of Japanese American individuals.

\begin{tabular}{|c|c|c|c|c|}
\hline Measure $^{a}$ & All Subjects $(\mathrm{N}=75)$ & HLD Cases $(\mathrm{N}=24)$ & Non-HLD $(\mathrm{N}=51)$ & $\mathrm{P}^{\mathrm{b}}$ \\
\hline Age (yrs) & $55.68 \pm 15.9$ & $66.51 \pm 10.65$ & $50.58 \pm 15.46$ & $1.23 \times 10^{-9}$ \\
\hline Sex (No. female, \%) & $40(53 \%)$ & $17(71 \%)$ & $23(45 \%)$ & 0.030 \\
\hline Diabetes WHO & 30 (41\%) & $13(54 \%)$ & $17(35 \%)$ & 0.129 \\
\hline Age at deafness (yrs) & - & $55.24 \pm 15.79$ & - & \\
\hline Ever smoked & $41(55 \%)$ & $19(79 \%)$ & $22(43 \%)$ & 0.001 \\
\hline Age began smoking (yrs) & $19.61 \pm 4.25$ & $19.05 \pm 4.55$ & $20.09 \pm 4.01$ & 0.334 \\
\hline Age stopped smoking (yrs) & $42.17 \pm 11.31$ & $42.33 \pm 11.78$ & $42.05 \pm 11.25$ & 0.907 \\
\hline Duration of smoking (yrs) & $11.47 \pm 14.08$ & $17.15 \pm 14.22$ & $9.2 \pm 13.51$ & 0.014 \\
\hline Packs-per-day & $0.53 \pm 0.71$ & $0.78 \pm 0.78$ & $0.42 \pm 0.65$ & 0.049 \\
\hline
\end{tabular}

${ }^{\mathrm{a}}$ Means \pm standard deviations of continuous measures (age, age of deafness, age began smoking, age stopped smoking, duration of cigarette smoking, packs-per-day) and the number and percentages of dichotomous variables (sex, diabetes status, cigarette ever-smoker status).

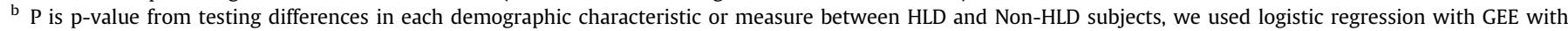
independence correlation structure to account for familial correlation.

Table 2

Demographic characteristics of Japanese American families.

\begin{tabular}{|c|c|c|c|c|c|c|c|c|c|}
\hline Characteristic & Average Per-Family & Family 1 & Family 2 & Family 3 & Family 4 & Family 5 & Family 6 & Family 7 & Family 8 \\
\hline $\mathrm{N}$ & $13 \pm 4$ & 10 & 9 & 16 & 10 & 10 & 10 & 19 & 13 \\
\hline HLD (N, \%) & $3 \pm 1$ & $4(40 \%)$ & $2(22 \%)$ & $4(25 \%)$ & $2(20 \%)$ & $2(20 \%)$ & $4(40 \%)$ & $2(11 \%)$ & $4(31 \%)$ \\
\hline Age (years) & $57.74 \pm 7.79$ & 66.75 & 64.95 & 53.46 & 58.21 & 53.90 & 67.31 & 49.77 & 47.57 \\
\hline Sex (\% male) & $43 \% \pm 10 \%$ & $40 \%$ & $33 \%$ & $38 \%$ & $40 \%$ & $50 \%$ & $30 \%$ & $58 \%$ & $54 \%$ \\
\hline Diabetes (\%) & $44 \% \pm 14 \%$ & $67 \%$ & $50 \%$ & $43 \%$ & $50 \%$ & $57 \%$ & $33 \%$ & $27 \%$ & $27 \%$ \\
\hline Age at deafness (yrs) & $53.96 \pm 6.46$ & 57.75 & 55.00 & 54.67 & 41.50 & 55.00 & 59.00 & 47.50 & 61.25 \\
\hline Ever smoked $^{\mathrm{b}}$ & $57 \% \pm 26 \%$ & $86 \%$ & $83 \%$ & $79 \%$ & $63 \%$ & $63 \%$ & $33 \%$ & $33 \%$ & $18 \%$ \\
\hline Age began smoking (yrs) & $20 \pm 1.57$ & 19.00 & 19.60 & 18.27 & 20.60 & 19.80 & 18.50 & 21.20 & 23.00 \\
\hline Age stopped smoking (yrs) & $42.22 \pm 5.79$ & 46.25 & 54.80 & 38.50 & 37.20 & 39.80 & 41.50 & 41.20 & 38.50 \\
\hline Duration of smoking (yrs) & $13.27 \pm 8.83$ & 23.00 & 29.33 & 13.82 & 10.38 & 12.50 & 7.67 & 6.67 & 2.82 \\
\hline Packs-per-day & $0.57 \pm 0.4$ & 1.17 & 0.75 & 0.58 & 0.31 & 1.07 & 0.25 & 0.40 & 0.05 \\
\hline
\end{tabular}

${ }^{a}$ Continuous covariates (age, age of deafness, age began smoking, age stopped smoking, duration of cigarette smoking, packs-per-day) were averaged within each family,

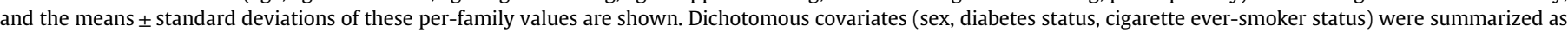
the percentage within each family, and the means \pm standard deviations of these per-family percentages are shown in this column of the table.

b Families are ordered in descending order based on \% cigarette ever smoker in family.

Table 3

Association between HLD risk and cigarette smoking.

\begin{tabular}{|c|c|c|}
\hline Cigarette Smoking exposure variable & Ever-smoker $\mathrm{OR}^{\mathrm{a}}\left(95 \% \mathrm{Cl}^{\mathrm{b}}\right) \mathrm{p}^{\mathrm{c}}$ & Packs-per-day $\mathrm{OR}^{\mathrm{d}}\left(95 \% \mathrm{CI}^{\mathrm{b}}\right), \mathrm{p}^{\mathrm{c}}$ \\
\hline No adjustment & $5.01(1.88,13.32) \mathrm{p}=0.001$ & $2.04(1.00,4.15) \mathrm{p}=0.049$ \\
\hline Model 1: Adjusted for age + sex + diabetes & $2.83(0.97,8.22) \mathrm{p}=0.056$ & $2.14(0.90,5.07) \mathrm{p}=0.084$ \\
\hline Model 2: Adjusted for age + sex + diabetes + smoking duration & $5.76(1.46,22.66) \mathrm{p}=0.012$ & $4.97(1.53,16.16) \mathrm{p}=0.008$ \\
\hline
\end{tabular}

a OR is odds ratio of having hearing loss disorder (HLD) in smokers compared to non-smokers. OR can also be interpreted as the multiplicative fold increase in risk of HLD comparing smokers to non-smokers.

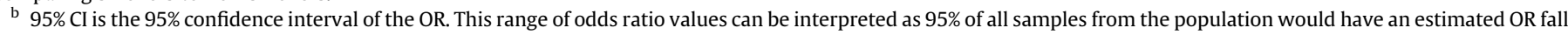
in this range.

c P-value corresponds to Wald's test; p-values $<0.05$ is evidence of significant smoking effects on risk of having hearing loss disorder (HLD).

d OR is odds ratio of of having HLD for every additional packs-per-day being smoked. OR can also be interpreted as the multiplicative fold increase in risk of HLD per each additional increase of packs per day smoked.

Further, we also identified three regions that appear to harbor HLD susceptibility genes, and these regions appear to be novel. We extend these linkage findings and provide the first evidence to our knowledge for gene $\times$ smoking interactions. Importantly, additional potential susceptibility regions for HLD appear to have been detected only after considering heterogeneous evidence of linkage based on smoking, in particular for the region on chromosome 8 between $66 \mathrm{Mb}$ and $107.3 \mathrm{Mb}$. These results demonstrate the importance of considering GxE even in the context of family and linkage based studies.

In agreement with others, our study results support an association between smoking and HLD, that remains significant after adjustment for age, gender, history of diabetes and duration of smoking. Similarly, our findings are consistent with others showing an elevated risk of HLD with ever smoking and with packs-per-day. For example, in a cross-sectional study Cruickshanks and colleagues (Cruickshanks et al., 1998) showed that current smokers have a $69 \%$ increased risk of developing hearing loss compared to non-smokers, adjusting for age, history of cardiovascular disease, alcohol consumption, occupational noise exposure, and education. Similarly, Helzner and colleagues (Helzner et al., 2005) demonstrated that current smokers have a 57\% increased risk of developing hearing loss compared to non-smokers through age-adjusted analyses. A cohort study conducted by Nakanishi and colleagues (Nakanishi et al., 2000) showed that the multivariate-adjusted risk for high-frequency hearing impairment was between 1.21 and 1.82 for current smokers, depending on the amount of cigarettes smoked per day. While our estimates are somewhat higher, it is possible that this may be due to uncontrolled confounding or possibly because we focused on families with multiple cases of HLD.

Results from the nonparametric linkage analysis indicated 
Table 4

Evidence of gene-by-environment interaction in subsets of families: Ordered subset analysis (OSA) results.

\begin{tabular}{|c|c|c|c|c|c|c|c|c|c|c|}
\hline $\begin{array}{l}\text { Smoking } \\
\text { covariate }\end{array}$ & CHR & $\begin{array}{l}\text { Location of OSA max } \\
\text { LOD }^{\mathrm{a}}(\mathrm{cM})\end{array}$ & $\begin{array}{l}\text { Marker at OSA } \\
\text { max LOD }\end{array}$ & $\begin{array}{l}\text { Location }^{\mathrm{b}} \text { (bp } \\
\text { build 37) }\end{array}$ & $\begin{array}{l}\text { Markers flanking 1-LOD } \\
\text { support interval }\end{array}$ & $\begin{array}{l}\text { 1-LOD support interval }{ }^{c} \\
\text { (bp, build 37) }\end{array}$ & $\begin{array}{l}\text { OSA Max } \\
\text { LOD }\end{array}$ & $\begin{array}{l}\text { Original } \\
\text { Max LOD }\end{array}$ & $\mathrm{p}$ & $\begin{array}{l}\text { No. } \\
\text { Families/ } \\
\text { Total }^{\mathrm{d}}\end{array}$ \\
\hline $\begin{array}{l}\text { Packs-per- } \\
\text { day }\end{array}$ & $8 q 21.3$ & 89 & D8S1119 & 87172020 & $\begin{array}{l}\text { D8S1136 } \\
\text { D8S1132 }\end{array}$ & $\begin{array}{l}66,053,167 \\
107,328,872\end{array}$ & 3.54 & 1.83 & 0.04 & $4 / 8$ \\
\hline \multirow[t]{5}{*}{$\begin{array}{l}\text { Ever } \\
\quad \text { smoking }\end{array}$} & 7p21.1 & 21.5 & D7S3051 & $18,284,528$ & $\begin{array}{l}\text { D7S1819 } \\
\text { D7S1802 }\end{array}$ & $\begin{array}{l}4,493,039 \\
20,705,304\end{array}$ & 3.3 & 1.77 & 0.04 & $2 / 8$ \\
\hline & $11 \mathrm{q} 23.1$ & 111.7 & D11S1986 & $111,223,605$ & $\begin{array}{l}\text { D11S2002 } \\
\text { D11S4464 }\end{array}$ & $\begin{array}{l}79,965,403 \\
123,626,627\end{array}$ & 2.1 & 1.12 & 0.03 & $2 / 8$ \\
\hline & $12 \mathrm{q} 32.1$ & 145.8 & D12S1045 & $130,397,7381$ lts & $\begin{array}{l}\text { D12S2078 } \\
\text { D12S392 }\end{array}$ & $\begin{array}{l}127,961,140 \\
130,607,434\end{array}$ & 2.6 & 1.15 & 0.01 & $3 / 8$ \\
\hline & $15 q 26.2$ & 96.4 & D15S816 & $95,019,784$ & $\begin{array}{l}\text { D15S1015 } \\
\text { D15S657 }\end{array}$ & $\begin{array}{l}67,839,381 \\
96,704,848\end{array}$ & 2.9 & 1.42 & 0.01 & $5 / 8$ \\
\hline & 20q13.3 & 92.2 & D20S171 & $57,808,030$ & D20S197 rs6089801 & $\begin{array}{l}46,160,764 \\
62,911,391\end{array}$ & 3 & 1.77 & 0.02 & $3 / 8$ \\
\hline
\end{tabular}

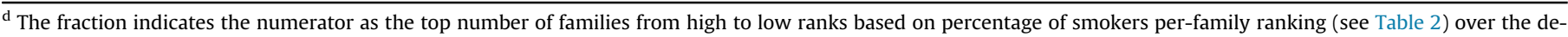
nominator which is the total number of 8 families. This subset of families was found to yield significant evidence gene-by-smoking interaction $(\mathrm{P}<0.05)$.

a Genetic location in centiMorgans of the max LOD for the given chromosome.

b Physical basepair positions (build 37) were determined by Rutgers Combined Linkage-Physical Map version 3 (Nato et al., 2018 ). If the marker was not found, the map position was extrapolated using a slope-intercept line equation and the positions of two nearby markers.

c 1-LOD support intervals were determined by using locations of the nearest markers closest to flanking positions with 1-LOD difference from the max LOD.

e There is no right-flanking marker beyond D20S171 on chromosome 20; therefore, the marker, rs6089801, with the highest base pair position on chromosome 20 in the Rutger's map (Matise et al., 2007) was used as the right-flanking marker.

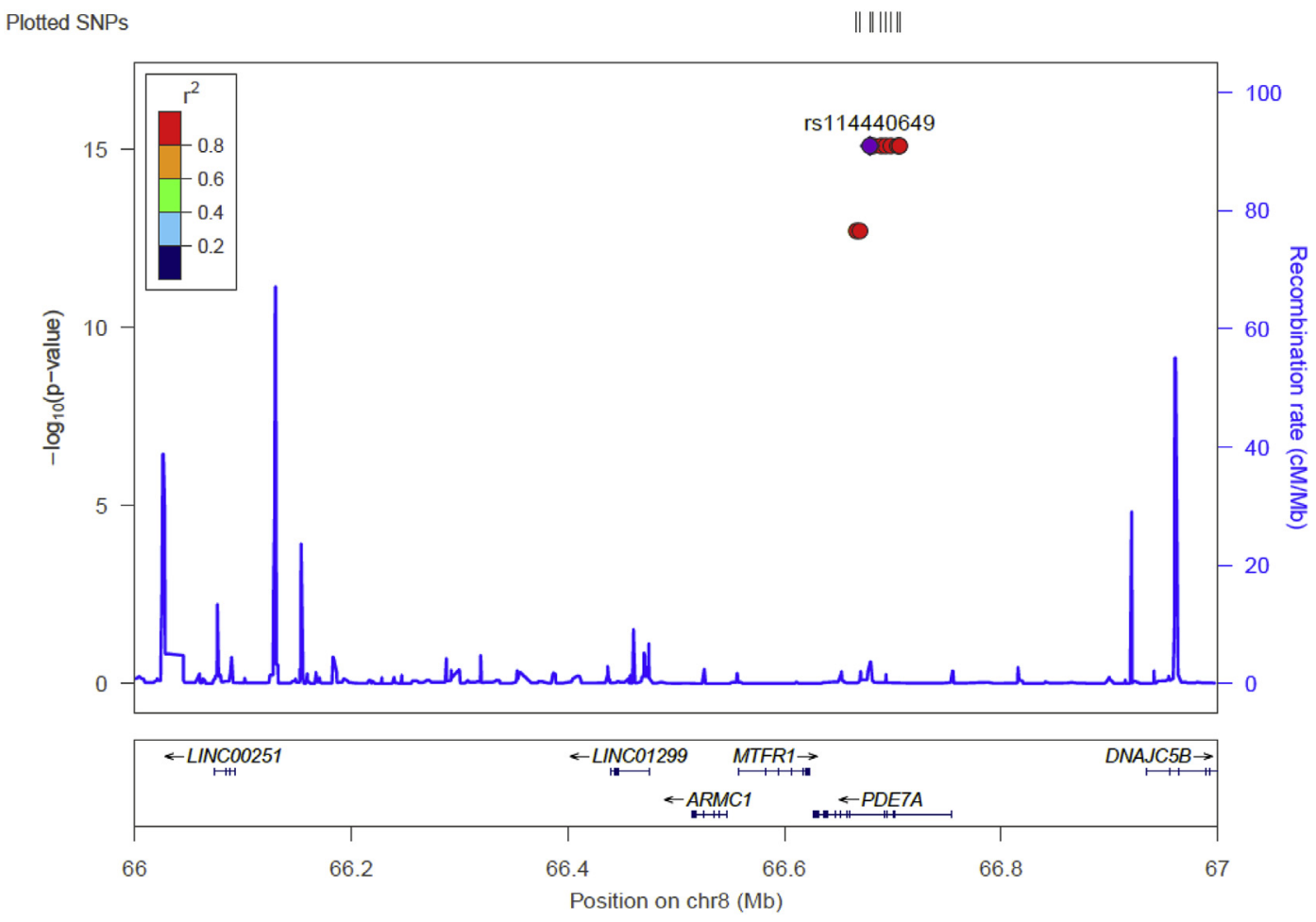

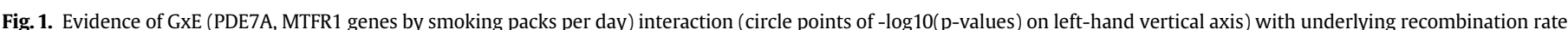

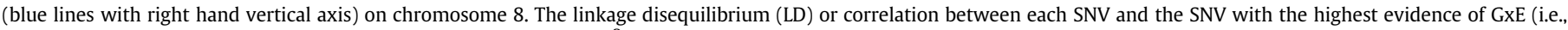

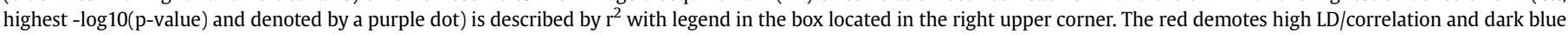

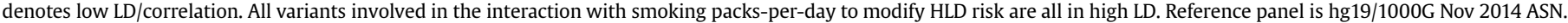
Plot was made using Locus Zoom (Prium et al., 2010).

suggestive evidence for linkage to putative HLD loci for three chromosomal regions: chromosomes 5p15, 8p23, and 17q21. Bykhovskaya et al. observed that a highly suggestive locus on chromosome 8 near D8S277, position 6,516,725 - 6,516,870, associated with progressive hearing loss in carriers of the A1555G mitochondrial mutation in Spanish, Italian, and Arab-Israeli families (Bykhovskaya et al., 2000). In the Arab-Israeli/Spanish/Italian families, the combined maximized allele-sharing LOD score was 3.1. Although there is evidence of linkage on chromosomes 8 and 17 in other studies, these nominated regions did not overlap with the regions of suggestive linkage in this study. Also, there are no reported HLD or related traits for the region on chromosome 5.

Moreover, results from the OSA indicated six novel genomic regions that appeared to be involved in gene-by-smoking 
interactions. Consequently, these regions on chromosomes 7, 8, 11, 12,15 and 20 did not contain any known HLD genes and appear to be novel regions for HLD. This is likely if genes in these regions only operate in the presence of smoking and are otherwise undetectable. These results are consistent with a recent large multi-ancestry genome-wide smoking interaction study of over 380,000 individuals showing that when considering interaction with smoking, 13 new loci associated with serum lipids were identified (Bentley et al., 2019). Aside from this recent study, there have been relatively few gene-environment-wide interaction studies (GEWIS) examining complex diseases Hutter et al. (2012); Cornelis et al. (2012), and even fewer that have addressed GxE in HLD. Studies that have addressed this have primarily focused on noise-induced hearing loss (NIHL). For instance, Carlsson and researchers (Carlsson et al., 2005) investigated whether genetic variability in the human antioxidant system is associated with high or low susceptibility to NIHL. However, no significant differences were found between susceptible and resistant groups. Yang and colleagues (Yang et al., 2006) determined whether there was an association of polymorphisms of the Hsp70 genes with susceptibility to NIHL among Chinese automobile workers. Their results suggested that some haplotypes of the Hsp70 gene cluster may be associated with a higher susceptibility to NIHL.

In particular, the variants of the PDE7A on chromosome 8 implicated in GxE interaction with smoking are also involved with the purine metabolism pathway. There is some evidence the purine metabolism pathway is involved with X-linked hearing loss with gene PRPS1 (Liu et al., 2013; Jimenez and Puig, 2012; Yang et al., 2017; Jinnah et al., 2013). This pathway is also correlated with smoking (Mouhamed et al., 2011; Jones et al., 2016). In addition, studies have also evaluated that hearing loss is twice as high among those with diabetes compared those without, and there is a $30 \%$ higher rate of hearing loss among those with pre-diabetes (Cullen and Cinnamond, 1993; Kim et al., 2017). Specifically, because GENNID families were selected based on multiple family members with T2D, and there is also evidence of uric acid being associated the MetS (Kushiyama et al., 2014), perhaps both MetS and hearing loss are mediated through an overlap of similar causal pathways, and the uric acid and purine metabolism is one of such pathways (Bainbridge et al., 2008; Simmonds et al., 1985; Kim et al., 2007). More studies will have to be performed to evaluate this hypothesis. In addition, although significant GxE variants were nominated with low p-values and account for multiple comparisons using a conservative Bonferroni correction, these findings should be considered suggestive until they are replicated and confirmed in independent samples.

There is increased motivation for the use of GEWIS or other methods that incorporate environmental factors when marginal effects of genetic factors are small and our results are the first to evaluate GxE in relation to HLD, but there were limitations of this study. First, HLD was self-reported. While the survey questioned whether they had been told by a healthcare worker that they had HLD, measured deafness using audiometric tests would have likely improved the accuracy of our phenotype. Furthermore, by not measuring hearing loss, it is likely that some cases with early hearing loss were missed. Our inability to subtype by severity also extends to our inability to subclassify or exclude known subtypes such as non-syndromic or syndromic status and by etiology (i.e., congenital, noise-induced). However, the resulting misclassification would likely result in a decrease in power to detect association, linkage, and GxE. Our approach in using a per family average definition of smoking status for the family unit may have also resulted in misclassification of the exposure and reduced the power to detect effects. Additionally, we did not have information on the participants' occupational exposure, including both ototoxic chemicals or excessive noise working environments and were unable to account for those potential confounders. Finally, it is possible that our results which were based on families selected for type 2 diabetes may not generalizable to families without diabetes.

\section{Conclusions}

Although, it has been estimated that over $16.8 \%$ of US adults were current smokers, defined as smoking 100 cigarettes or more during one's lifetime and currently smoking every day or some days (Center for Disease Control and Prevention, 2015), differences in smoking patterns among the Japanese American population may differentially affect the risk of developing HLD. Thus, clarification of the association between smoking and HLD may have a large impact on the public health and help motivate significant behavioral change among at-risk adults.

\section{Funding}

This study was funded by the National Institutes of Health grant NIH R01 HL113189 (Edwards KL, Principal Investigator) 2/1/2014-3/ 31/2017 Identifying Genes Underlying Linkage Peaks for Clusters of CVD Risk Factors in a Multi-Ethnic Family Study and also funded by a grant (7-05-RA-34) from the American Diabetes Association (Edwards KL, Principal Investigator).

\section{CRediT authorship contribution statement}

Jia Y. Wan: Data curation, Formal analysis, Writing - original draft, Writing - review \& editing. Christina Cataby: Writing original draft, Writing - review \& editing. Andrew Liem: Writing original draft, Writing - review \& editing. Emily Jeffrey: Writing original draft, Writing - review \& editing. Trina M. NordenKrichmar: Formal analysis, Writing - review \& editing. Deborah Goodman: Writing - review \& editing. Stephanie A. Santorico: Writing - original draft, Writing - review \& editing. Karen L. Edwards: Funding acquisition, Conceptualization, Methodology, Writing - original draft, Writing - review \& editing.

\section{Acknowledgements}

American Diabetes Association GENNID Study Group: Genetic material was collected and families characterized by Eric Boerwinkle, PhD, University of Texas Health Science Center; John Buse, MD, PhD, University of North Carolina; Ralph DeFronzo, MD, University of Texas Health Science Center; David Ehrmann, MD, University of Chicago; Steven C. Elbein, MD, University of Utah/ University of Arkansas; Wilfred Fujimoto, MD, and Steven E. Kahn, $\mathrm{MB}, \mathrm{ChB}$, University of Washington; Craig L. Hanis, PhD, University of Texas Health Science Center; Richard A. Mulivor, PhD, and Jeanne C. Beck, PhD, Coriell Cell Repositories; Jill Norris, PhD, University of Colorado School of Medicine; M. Alan Permutt, MD, and Philip Behn, MD, Washington University School of Medicine; Leslie Raffel, MD, Cedars-Sinai Medical Center; and David C. Robbins, MD, Medlantic Research Institute. Genotyping services were also provided by the Northwest Genomics Center (NWGC).

\section{Appendix A. Supplementary data}

Supplementary data to this article can be found online at https://doi.org/10.1016/j.heares.2019.107875.

\section{References}

Abecasis, G.R., Cherny, S.S., Cookson, W.O., Cardon, L.R., 2002. Merlin-rapid analysis 
of dense genetic maps using sparse gene flow trees. Nat. Genet. 30, 97-101. https://doi.org/10.1038/ng786.

Agrawal, Y., Platz, E.A., Niparko, J., 2008. Prevalence of hearing loss and differences by demographic characteristics among US adults: data from the National Health and Nutrition Examination Survey, 1999-2004. Arch. Intern. Med. 168, $1522-1530$.

Bainbridge, K.E., Hoffman, H.J., Cowie, C.C., 2008. Diabetes and hearing impairment in the United States: audiometric evidence from the national health and nutrition examination surveys, 1999-2004. Ann. Intern. Med. 149, 1-10.

Bentley, A.R., Sung, Y.J., Brown, M.R., Winkler, T.W., Kraja, A.T., et al., 2019. Multiancestry genome-wide gene-smoking interaction study of 387,272 individuals identifies new loci associated with serum lipids. Nat. Genet. 51, 636-648. https://doi.org/10.1038/s41588-019-0378-y.

Besag, J., Clifford, P., 1991. No TitleSequential Monte Carlo p-values. Biometrika 78, 301-304.

Browning, B.L., 2006. FLOSS: flexible ordered subset analysis for linkage mapping of complex traits. Bioinformatics 22, 512-513. https://doi.org/10.1093/bioinformatics/btk012.

Bykhovskaya, Y., Estivill, X., Taylor, K., et al., 2000. Candidate locus for a nuclear modifier gene for maternally inherited deafness. Am. J. Hum. Genet. 66, 1905-1910. https://doi.org/10.1086/302914.

Carey, V.J., 2015. Ported to R by TL (versions 3. 1. and 4. 4. and BR (version 4. 13. gee: generalized Estimation Equation Solver. R package version 4.13-19. Published. https://cran.r-project.org/package=gee.

Carlsson, P.-I., Van Laer, L., Borg, E., et al., 2005. The influence of genetic variation in oxidative stress genes on human noise susceptibility. Hear. Res. 202, 87-96. https://doi.org/10.1016/j.heares.2004.09.005.

Center for Disease Control and Prevention, 2015. Current cigarette smoking among adults-United States, 2005-2014. Morb. Mortal. Wkly. Rep. 64, 1233-1240.

Chen, M.-H., Yang, Q., 2015. GWAF: genome-wide association/interaction analysis and rare variant analysis with family data. $\mathrm{R}$ package version 2.2.n. https:// CRAN.R-project.org/package $=$ GWAF.

Cornelis, M.C., Tchetgen, E.J.T., Liang, L., et al., 2012. Gene-environment interactions in genome-wide association studies: a comparative study of tests applied to empirical studies of type 2 diabetes. Am. J. Epidemiol. 175, 191-202. https:// doi.org/10.1093/aje/kwr368.

Cruickshanks, K.J., Klein, R., Klein, B.E., Wiley, T.L., Nondahl, D.M., Tweed, T.S., 1998 Cigarette Smoking and Hearing Loss: the Epidemiology of Hearing Loss Study, vol. 279, pp. 1715-1719. https://doi.org/10.1001/jama.279.21.1715.

Cullen Jr., Cinnamond, M.J., 1993. Hearing loss in diabetics. J. Laryngol. Otol. 107, 179-182. PMID:8509689.

Dickson, S.P., Wang, K., Krantz, I., Hakonarson, H., Goldstein, D.B., 2010. Rare variants create synthetic genome-wide associations. PLoS Biol. 8, e1000294 https:/ doi.org/10.1371/journal.pbio.1000294.

Edwards, K.L., Hutter, C.M., Wan, J.Y., Kim, H., Monks, S.A., 2008. Genome-wide linkage scan for the metabolic syndrome: the GENNID study. Obesity 16 1596-1601. https://doi.org/10.1038/oby.2008.236.

Ehm, M.G., Karnoub, M.C., Sakul, H., et al., 2000. Genomewide search for type 2 diabetes susceptibility genes in four American populations. Am. J. Hum. Genet 66, 1871-1881. https://doi.org/10.1086/302950.

Friedman, R.A., Van Laer, L., Huentelman, M.J., et al., 2009. GRM7 variants confer susceptibility to age-related hearing impairment. Hum. Mol. Genet. 18, 785-796. https://doi.org/10.1093/hmg/ddn402.

Hancock, D.B., Martin, E.R., Li, Y.J., Scott, W.K., 2007. Methods for interaction analyses using family-based case-control data: conditional logistic regression versus generalized estimating equations. Genet. Epidemiol. 31, 883-893. https://doi.org/10.1002/gepi.20249.

Hauser, E.R., Watanabe, R.M., Duren, W.L., Bass, M.P., Langefeld, C.D., Boehnke, M., 2004. Ordered subset analysis in genetic linkage mapping of complex traits. Genet. Epidemiol. 27, 53-63. https://doi.org/10.1002/gepi.20000.

Helzner, E.P., Cauley, J.A., Pratt, S.R., et al., 2005. Race and sex differences in agerelated hearing loss: the health, aging and body composition study. J. Am. Geriatr. Soc. 53, 2119-2127. https://doi.org/10.1111/j.1532-5415.2005.00525.x.

Hilgert, N., Smith, R.J.H., Van Camp, G., 2009. Forty-six genes causing nonsyndromic hearing impairment: which ones should be analyzed in DNA diagnostics? Mutat. Res. 681, 189-196. https://doi.org/10.1016/j.mrrev.2008.08.002.

Hinrichs, A.S., Karolchik, D., Baertsch, R., Barber, G.P., Bejerano, G., et al., 2006. The UCSC genome browser database: update 2006. Nucleic Acids Res. 1 (34), D590-D598 (Database issue).

Horikawa, C., Kodama, S., Tanaka, S., Fujhara, K., Hirasawa, R., et al., 2013. Diabetes and risk of hearing impairment in adults: a meta-analysis. J. Clin. Endocrinol. Metab. 981, 51-58.

Hu, H., Sasaki, N., Ogasawara, T., Nagahama, S., Akter, S., Kuwahara, K., Kochi, T., Eguhi, M., et al., 2019. Smoking, smoking cessation, and the risk of hearing loss: Japan epidemiology collaboration on occupational health study. Nicotine Tob. Res. 21, 481-488.

Hutter, C.M., Chang-Claude, J., Slattery, M.L., et al., 2012. Characterization of geneenvironment interactions for colorectal cancer susceptibility loci. Cancer Res. 72, 2036-2044. https://doi.org/10.1158/0008-5472.CAN-11-4067.

Huyghe, J.R., Van Laer, L., Hendrickx, J.-J., et al., 2008. Genome-wide SNP-based linkage scan identifies a locus on 8q24 for an age-related hearing impairment trait. Am. J. Hum. Genet. 83, 401-407. https://doi.org/10.1016 j.ajhg.2008.08.002.

Ito, T., Noguchi, Y., Yashima, T., Ohno, K., Kitamura, K., 2010. Hereditary hearing loss and deafness genes in Japan. J. Med. Dent. Sci. 57, 1-10. PMID 20437760.
Jimenez, R.T., Puig, J.G., 2012. Purine metabolism in the pathogenesis of hyperuricemia and inborn errors of purine metabolism associated with disease. Gout Other Cryst. Arthropathies 36-50.

Jinnah, H.A., Savina, R.L., Van Den Berghe, G., 2013. Metabolic disorders of purine metabolism affecting the nervous system. Handb. Clin. Neurol. 113, 1827-1836. https://doi.org/10.1016/B978-0-444-59565-2.00052-6.

Jones, D.P., Walker, D.I., Uppal, K., Rohrbek, P., Mallon, T.M., Go, Y.M., 2016. Metabolic pathways and networks associated with tobacco use in military personnel. J. Occup. Environ. Med. 58, S111-S116. https://doi.org/10.1097/ JOM.0000000000000763.

Karlsmose, B., Lauritzen, T., Engberg, M., Parving, A., 2000. A five-year longitudinal study of hearing in a Danish rural population aged 31-50 years. Br. J. Audiol. 34, 47-55. http://www.ncbi.nlm.nih.gov/pubmed/10759077. (Accessed 21 April 2016).

Kim, H.J., Sohn, K.M., Shy, M.E., Krajewski, K.M., et al., 2007. Mutations in PRPS1, which encodes the phosphoribosyl pyrophosphate synthetase enzyme critical for nucleotide biosynthesis, cause hereditary peripheral neuropathy with hearing loss and optic neuropathy (CMTX5). Am. J. Hum. Genet. 81, 552-558. https://doi.org/10.1086/519529.

Kim, M.B., Zhang, Y., Chang, Y., Ryu, S., Choi, Y., et al., 2017. Diabetes mellitus and the incidence of hearing loss: a cohort study. Int. J. Epidemiol. 46, 717-726. https:// doi.org/10.1093/ije/dyw342.

Kobayashi, M., Miyagawa, M., Nishio, S., Moteki, H., Fujikawa, T. et al., 2018. WFS1 Mutation screening in a large series of Japanese hearing loss patients: massively parallel DNA sequencing based analysis. PLoS One 13, e0193359.

Kong, A., Cox, N.J., 1967. Allele-sharing models: LOD scores and accurate linkage tests. Am. J. Hum. Genet. 61, 1179-1188. https://doi.org/10.1086/301592.

Kushiyama, A., Tanaka, K., Hara, S., Kawazu, S., 2014. Linking uric acid metabolism to diabetic complications. World J. Diabetes 5, 787-795. https://doi.org/10.4239/ wjd.v5.i6.787.

Kvestad, E., Czajkowski, N., Krog, N.H., Engdahl, B., Tambs, K., 2012. Heritability of hearing loss. Epidemiology 23, 328-331. https://doi.org/10.1097/ EDE.0b013e318245996e.

Lavinsky, J., Crow, A.L., Pan, C., et al., 2015. Genome-wide association study identifies nox3 as a critical gene for susceptibility to noise-induced hearing loss. PLoS Genet. 1, e1005094 https://doi.org/10.1371/journal.pgen.1005094.

Lin, F.R., Niparko, J.K., Ferrucci, L., 2011a. Hearing loss prevalence in the United States. Arch. Intern. Med. 171, 1851-1852.

Lin, F.R., Thorpe, R., Gordon-Salant, S., Ferrucci, L., 2011b. Hearing loss prevalence and risk factors among older adults in the United States. J. Gerontol. A Biol. Sci. Med. Sci. 66, 582-590. https://doi.org/10.1097/EDE.0b013e318245996e.

Liu, X.Z., Xie, D., Yuan, H.J., de Brouwer, A.P.M., Christodoulou, J., Yan, D., 2013. Hearing loss and PRPS1 mutations: wide spectrum of phenotypes and potential therapy. Int. J. Audiol. 52, 23-28. https://doi.org/10.3109/ 14992027.2012 .736032$.

Matise, T.C., et al., 2007. A second-generation combined linkage physical map of the human genome. Genome Res. 17, 1783-1786. PMID: 17989245 PMCID: PMC2099587.

McMahon, C.M., Kifley, A., Rochtchina, E., Newall, P., Mitchell, P., 2008. The contribution of family history to hearing loss in an older population. Ear Hear. 29, 578-584. https://doi.org/10.1097/EDE.0b013e318245996e.

Mayo Clinic, 2015. Hearing loss risk factors. http://www.mayoclinic.org/diseasesconditions/hearing-loss/basics/risk-factors/con-20027684. (Accessed February 2016)

Mohr, P.E., Feldman, J.J., Dunbar, J.L., et al., 2000. The societal costs of severe to profound hearing loss in the United States. Int. J. Technol. Assess. Health Care $16,1120-1135$

Mouhamed, D.H., Ezaher, A., Meffati, F., Douki, W., Gaha, l., Najjar, M.F., 2011. Effect of cigarette smoking on plasma uric acid concentrations. Environ. Health Prev. Med. 16, 307-312. https://doi.org/10.1007/s12199-010-0198-2.

Nakanishi, N., Okamoto, M., Nakamura, K., Suzuki, K., Tatara, K., 2000. Cigarette smoking and risk for hearing impairment: a longitudinal study in Japanese male office workers. J. Occup. Environ. Med. 42, 1045-1049. https://doi.org/ 10.1097/00043764-200011000-00001.

Nato, A.Q., Buyske, S., Matise, T.C., 2018. The Rutgers map: a third-generation combined linkage-physical map of the human genome. Human genetics institute of New Jersey second Research day. Life sciences building. Rutgers University, Piscataway, NJ, USA. website. http://compgen.rutgers.edu/rutgers maps.shtml.

Ng, S.B., Turner, E.H., Robertson, P.D., Fluygare, S.D., Bigham, A.W., et al., 2009. Targeted capture and massively parallel sequencing of 12 human exomes. Nature 461 (7261), 272-276.

Nomura, K., Nakao, M., Morimoto, T., 2005. Effect of smoking on hearing loss: quality assessment and meta-analysis. Prev. Med. 40, 138-144.

O'Neill, M., 1996. A gene for autosomal dominant late-onset progressive nonsyndromic hearing loss, DFNA10, maps to chromosome 6. Hum. Mol. Genet. 5, 853-856. https://doi.org/10.1093/hmg/5.6.853.

Pruim, R.J., Welch, R.P., Sanna, S., Teslovich, T.M., Chines, P.S., Gliedt, T.P., Boehnke, M., Abecasis, G.R., Willer, C.J., 2010. LocusZoom: regional visualization of genome-wide association scan results. Bioinformatics 26 (18), 2336-2337, 2010 September 15

Raffel, L.J., Robbins, D.C., Norris, J.M., et al., 1996. The GENNID Study. A resource for mapping the genes that cause NIDDM. Diabetes Care 19, 864-872. http://www. ncbi.nlm.nih.gov/pubmed/8842605. (Accessed 1 February 2016).

R Core Team, 2019. R: A Language and Environment for Statistical Computing. R 
Foundation for Statistical Computing, Vienna, Austria. URL https://www.Rproject.org/.

Rohayem, J., Ehlers, C., Wiedeman, B., Holl, R., Oexle, K., et al., 2011. Diabetes and neurodegeneration in Wolfram syndrome. Diabetes Care 34, 1503-1510. https://doi.org/10.2337/dc10-1937.

Shearer, A.E., Hildebrand, M.S., Smith, R.J.H., 2014. Deafness and hereditary hearing loss overview. http://www.ncbi.nlm.nih.gov/books/NBK1434/. (Accessed 4 February 2016).

Siegelaub, A.B., Friedman, G.D., Adour, K., Seltzer, C.C., 1974. Hearing loss in adults. Arch. Environ. Health Int. J. 29, 107-109.

Simmonds, H.A., Webster, D.R., Lingam, S., Wilson, J., 1985. An inborn error of purine metabolism, deafness and neurodevelopmental abnormality. Neuropediatrics 16, 106-108. https://doi.org/10.1055/s-2008-1052552.

Smith, R.J., Shearer, A.E., Hildebrand, M.S., Camp, G.Van, January 2014. Deafness and Hereditary Hearing Loss Overview. http://www.ncbi.nlm.nih.gov/books/ NBK1434/.

Team R.C, 2015. R: a language and environment for statistical computing. Published. https://www.r-project.org/.
Uchida, Y., Sugiura, S., Nakashima, T., Ando, F., Shimokata, H., 2012. Estimates of the size of the hearing-impaired elderly population in Japan and 10-year incidence of hearing loss by age, based on data from the National Institute for Longevity Sciences-Longitudinal Study of Aging (NILS-LSA). Nihon Ronen Igakkai Zasshi 49, 222-227.

Whittemore, A.S., Halpern, J., 1994. A class of tests for linkage using affected pedigree members. Biometrics 50, 118-127. http://www.ncbi.nlm.nih.gov/ pubmed/8086596. (Accessed 19 February 2016).

WHO, 2006. Definition and diagnosis of diabetes mellitus and intermediate hyperglycaemia. http://www.who.int/diabetes/publications/diagnosis diabetes2006/en/. (Accessed 18 April 2016).

Yang, H.-F., Kao, T.-W., Peng, T.-C., Sun, Y.-S., Liaw, F.-Y., Want, C.-C., et al., 2017. Serum uric acid relation for hearing threshold shift. Clin. Exp. Otorhinolaryngol. 10, 143-147. https://doi.org/10.21053/ceo.2016.00346.

Yang, M., Tan, H., Yang, Q., et al., 2006. Association of hsp70 polymorphisms with risk of noise-induced hearing loss in Chinese automobile workers. Cell Stress Chaperones 11, 233-239. http://www.ncbi.nlm.nih.gov/pubmed/17009596. 